\title{
Disease, Duration and Death
}

\author{
Narayan Bahadur Basnet* \\ Children's Medical Diagnosis Center (CMDC), PO Box 1563, Chabahil, Kathmandu, Nepal \\ *Corresponding author: Narayan Bahadur Basnet, Children's Medical Diagnosis Center (CMDC), PO Box 1563, Chabahil, Kathmandu, Nepal
}

Received: April 10, 2020; Accepted: July 06, 2020; Published: July 13, 2020

Life has always been threaten by diseases, calamities, catastrophes leading to death caused by various known or unknown, animate or inanimate objects in human's relatively medium life span. Ever since the documentation of the human history, it is well known that man loved their body and prefer to live in accordance with their wishes. When rationale judgment became prominent after the experiences and observations of life and death events, they started searching remedies such as medicine. This is how medicine evolved since our early civilization. With the development of reason, logic, observation, experimentation and practical application, we learned tremendous ways of saving body, brain and behavior. However, as time passes human environment changes unpredictably leading to change in human behavior and attitude towards objects/materials and living beings. It is not only a matter of physical, biological or cosmic change but also behavior of everything that brought unprecedented events such as unexpected war, epidemic, catastrophes etc. leading to death $[1,2]$. Measurement of several physical parameters of human and universal bodies has become routine but various functions/characters in relation to time has yet to measure fully. This is the point we fall short to save humans promptly resulting high number of unexpected loss of life such as in COVID-19 pandemic. Among 1554960 covid-19 infected population in more than 209 countries, territories and two conveyances $5.9 \%$ died, and among the deaths more than $80 \%$ occurring in just 10 countries (USA, Spain, Italy, Germany, France, China, Iran, UK, Belgium, Netherlands) of the world in the last three months duration [2].

Disease is an abnormal architecture/anatomy, function, condition of the body and mind in a specific duration. Many times and circumstances death occurs due to unprecedented cause, behavior or ignorance. Therefore, it is essential to know the unknown environment and diverse nature and behavior of human beings to diagnose epidemicity of the disease. Despite vast scientific discoveries and new achievement, there is a big hole in the measurement of core human behavior and intelligence. Human body, intelligence and behavior plays a great role in the defense mechanism as well as association in the causation, development, cessation of disease in specific duration in specific place/s. So far we are devoid of the precise knowledge on the creation of covid-19 however many scientists have been trying to explore the mystery of the occurrences, nature and impact on the human population of the globe [3].
The duration or natural course of illness or diseases is important in the management of cases, carrier as well as prevention of complications and death [4]. Alert researchers identify the key factors of the disease when there is sudden rise of cases of similar features in a short period. Ignorance about the nature of pathogen and ignorance of the general population about the disease leads to higher number of deaths in a very short duration. Lack of alertness in changing behavior and environment of the disease in the population further complicates its management and increases the number of deaths. The challenge of the new disease, ignorance on the part of environment and human behavior help to expand disease dimensions in terms of time, place and person.

Opportunities such as chance, experience, observation and experimentation lead to discovery and development of medicine and care system that can make our life easier, comfortable and lengthier. This is the beauty of medical discipline, research and practice in human population. A dynamic patience where a body and brain searches a remedy continuously in response to disease is probably the best stimulus to initiate new knowledge, skills, practice to cure patient and prevent death. Lack of precise knowledge of duration and the nature of the disease is biggest obstacles in managing covid-19 at present and many more diseases that are possible in the future. Following the spread of disease and management of the patient (source) meticulously in global environment, recording the evidences and continuous sharing among the fellow researchers and responsible individuals are the most important aspects of pandemic control.

Alertness, continuous searches, dynamic patience can help humans to increase its capacity to deal with covid-19 pandemic. Change in seasonality in different geographical regions may affect duration of the diseases and distribution of death in humans. This demands thinking globally and acting globally.

Keywords: Covid-19, Death, Disease, Duration, Pandemic

\section{References}

1. Riedel S (2004) Biological warfare and bioterrorism: a historical review. BUMC Proceedings 17: 400-406. [crossref]

2. Covid-19 Coronavirus Pandemic, Worldometer. Accessed on April 09, 2020, 16:30 GMT.

3. Zhou P, Yang X, Wang X, Hu B, Zhang L, et al. (2020) A pneumonia outbreak associated with a new coronavirus of probable bat origin. Nature 579: 270-273. [crossref]

4. Rothan HA, Byrareddy SN (2020) The epidemiology and pathogenesis of coronavirus disease (COVID-19) outbreak. Journal of Autoimmunity 109: 102433. [crossref]

Citation:

Narayan Bahadur Basnet (2020) Disease, Duration and Death. Internal Med Res Open J Volume 5(3): 1-1. 